\title{
The 'Interactive' of Interactive Storytelling: Customizing the Gaming Experience
}

\author{
Barbaros Bostan $^{1}$ and Tim Marsh ${ }^{2}$ \\ ${ }^{1}$ Interactive and Digital Media Institute (IDMI), National University of Singapore, Level 2, \\ I-Cube Building No.21, Heng Mui Keng Terrace, 119613, Singapore \\ idmbb@nus.edu.sg \\ ${ }^{2}$ Faculty of Arts \& Social Sciences, National University of Singapore, Blk AS6, 11 \\ Computing Drive, 117416, Singapore
}

\begin{abstract}
In this article, we define interactive storytelling as a gaming experience where the form and content of the game is customized in real time and tailored to the preferences and needs of the player to maximixe enjoyment. The primary focus of interactive storytelling should not be on the attributes of the technology or characteristics of the medium, such as the AI techniques, planning formalisms, story representations, etc. but on different interaction levels provided by computer games and basic components of player enjoyment such as difficulty levels and gaming rewards. In conducting an analysis of interactive storytelling systems, we propose a user-centered approach to interactive storytelling by defining different customization levels for an optimum gaming experience.
\end{abstract}

Keywords: Computer games, interactivity, interactive storytelling, gaming experience, user-centered design.

\section{Interactive Storytelling: Beyond Story Structures}

Interactive storytelling is a gaming experience where the form and content of the game is modified in real time and tailored to the preferences and needs of the player to provide a sense of control over the mutual discourse of play. After all, the fundamental goal of interactivity is to allow the user different choices to be able to receive a highly personalized end result [1]. From our point of view, player modelling or profiling is the first step in dynamic reconfiguration of the virtual world to provide personalized gaming experiences. And the second step is in the player-centered approach to interactive storytelling is to decide on the parameters to be adapted or reconfigured beyond story plots and artificial intelligence capabilities of virtual agents. The term reconfiguration is transforming the "push button" interactivity into the productive process of gameplay where the user makes significant interventions into a game world to produce dynamic effects throughout the system [2]. Given below is the figure showing possible customization levels for computer games and our proposed customization levels for interactive storytelling systems. 


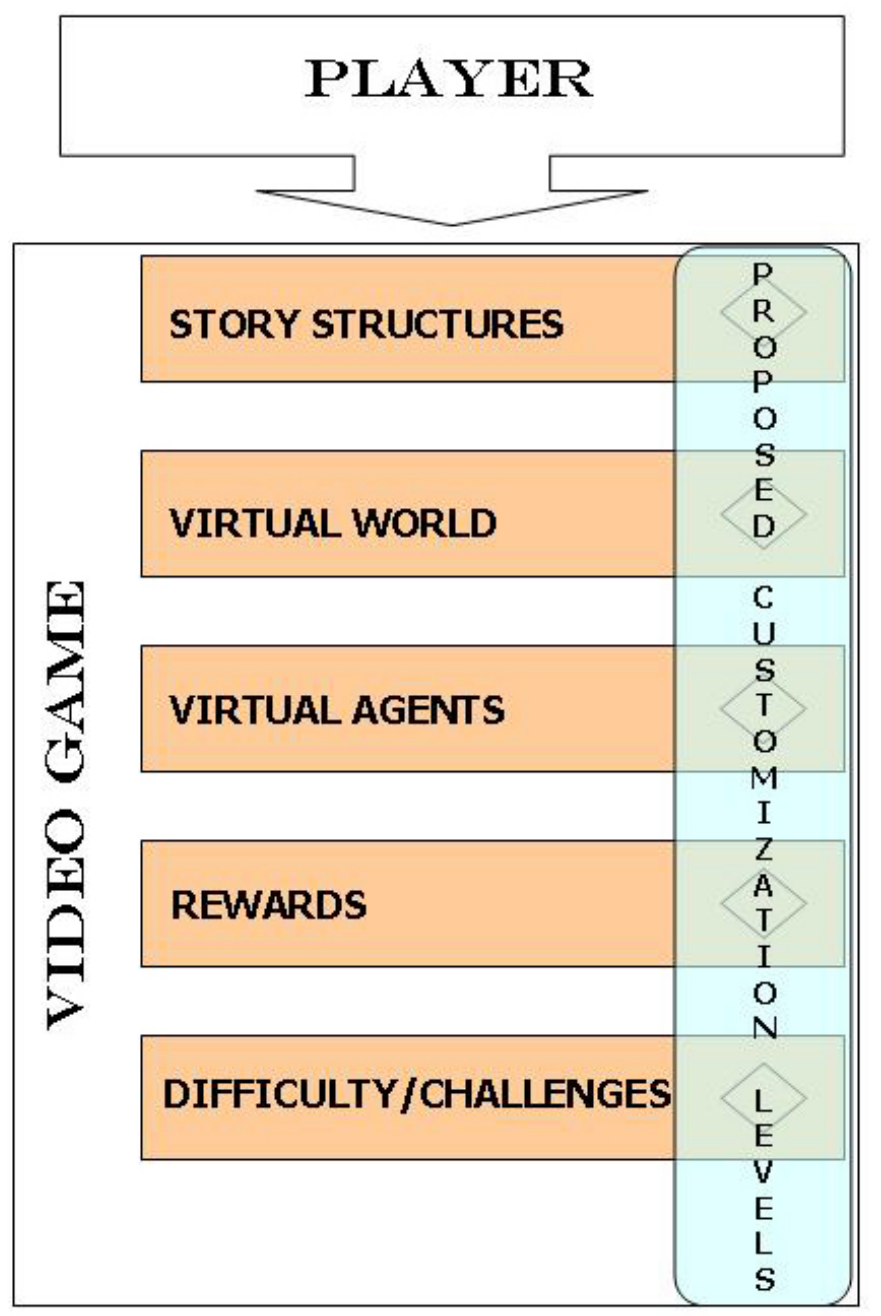

Fig. 1. Proposed customization levels for interactive storytelling systems

Customization of the gaming experience requires constant monitoring of the player actions within the virtual world. In this regard, Marsh et al. [3] proposed an approach to continuously and unobtrusively gather data about player interactions and a method to analyze and identify player behaviour, patterns and experience [4,5,6]. Player actions shall give an idea about his/her preferences or needs, but it should not be forgotten that the restrictions imposed by the game mechanics significantly reduce the number of player needs satisfied by a game. We claim that gaming experience should be customized for different interaction levels discussed in this study. Beyond story structures, the system should be capable of making real-time modifications on the virtual world (physical appearance, geography, regional history, life and society) and the virtual characters, as well as the player's avatar (physical appearance, personality, 
relations with other characters, traits, motives). Two other customization levels for interactive storytelling systems are the rewards given (experience points, items, spells, titles, guild ranks) and the difficulty levels provided. Having defined these five customization levels (story structures, virtual world, virtual agents, rewards, difficulty), it is important to note that most of the commercial games focus on customizing story structures by player alignment (evil-neutral-good) and most of the research projects focus on customizing story structures by play styles. We claim that gaming experience should be customized on all these levels according to the preferences and needs of the player.

Although the majority of the interactive storytelling systems focus on the capabilities of the drama manager or the story planning processes, we believe that the "interactive" part of interactive storytelling is the user modelling or the player profiling that handles player interactions and defines how the experience should be customized for the player. Based on play styles, character classes or player types, the drama managers usually customize the story plots or NPC goals. But the critical questions are "what" to customize, "how" to customize and "why" to customize. Are story plots or character interrelations the only elements to be customized to provide an interactive gaming experience? This article already defined five different customization levels (story structures, virtual world, virtual agents, rewards, difficulty) for optimizing the gaming experience. And why is there a need for customization? In this regard, it should not be forgotten that motivation to reach a goal is influenced by both personal factors (needs, motivations, and goals) and situational factors (opportunities and possible incentives provided by the environment). Thus, we believe that gameplaying experience should be customized for maximizing player enjoyment or providing an optimum gaming experience which requires an understanding of player's psychology, especially his/her needs, motivations, and goals.

Are the play styles or player types in the literature the only way to portray player preferences? Although there have been attempts to define the basic motivations behind game playing, different approaches to motivational aspects of player behavior, when applied to computer games with different structures and content, represent different facets of player psychology and define several variables that leave much scope for subjective interpretation. In this regard, the motivational variables defined by the major studies in literature are more likely to shed light on the popular question "why do people play computer games," rather than predicting player behaviour or understanding player interactions/choices in a computer game. In order to define 'how' to customize the gaming experience, the motivational framework proposed by Bostan [7] should provide better insights since the study is based on psychological needs and analyzes these needs in relation to the gaming situations of a computer game. In an attempt to take this study one step further and to identify the common interaction patterns between these individual needs, Bostan and Kaplancali [8] applied the same motivational framework to another computer game and analyzed psychological needs by defining the driving game mechanics behind them. The same authors also analyzed user-created content (mods) of a popular computer game within the same motivational framework in terms of the needs they satisfy [9]. For future studies, this motivational framework should provide a convenient way of understanding player preferences. 


\section{References}

1. Sundar, S.S.: Social psychology of interactivity in human-website interaction. In: Joinson, A.N., McKenna, K.Y.A., Postmes, T., Rieps, U.D. (eds.) The Oxford Handbook of Internet Psychology, pp. 89-102. Oxford University Press, Oxford (2007)

2. Dovey, J., Kennedy, H.W.: Game Cultures: Computer Games as New Media. Open University Press, Maidenhead \& Milton Keynes (2006)

3. Marsh, T., Yang, K., Shahabi, C., Wong, W.L., Nocera, L., Carriazo, E., Kyriakakis, C.: Automating the Detection of Breaks in Continuous User Experience with Computer Games. In: Proceedings of CHI 2005 Human Factors in Computing Systems, Extended Abstracts, Portland, USA. ACM Press, New York (2005)

4. Yang, K., Marsh, T., Mun, M., Shahabi, C.: Continuous Archival and Analysis of User Data in Virtual and Immersive Game Environments. In: The 2nd ACM Workshop on Capture, Archival and Retrieval of Personal Experiences (CARPE 2005), Singapore (2005)

5. Marsh, T., Yang, K., Shahabi, C.: Game development for experience through staying there. In: ACM SIGGRAPH Video Games Symposium, Boston, USA. ACM Press, New York (2006)

6. Marsh, T., Smith, S., Yang, K., Shahabi, C.: Continuous and Unobtrusive Capture of UserPlayer Behaviour and Experience to Assess and Inform Game Design and Development. In: 1st World Conference for Fun 'n Games, UK (2006)

7. Bostan, B.: Player Motivations: A Psychological Perspective. ACM Computers in Entertainment 7(2) (2009)

8. Bostan, B., Kaplancali, U.: Explorations in Player Motivations: Game Mechanics. In: Proceedings of GAMEON 2009, Düsseldorf, Germany (2009)

9. Bostan, B., Kaplancali, U.: Explorations in Player Motivations: Game Mods. In: Proceedings of GAMEON-ASIA 2010, Shanghai, China (2010) 